\title{
EL MOVIMIENTO FEMINISTA Y LA CONSTRUCCIÓN DE MARCOS DE INTERPRETACIÓN El caso de la violencia contra las mujeres
}

\begin{abstract}
ANA DE MIGUEL ÁLVAREZ
Universidad de A Coruña

\section{PALABRAS CLAVE ADICIONALES}

Movimientos Sociales, Género, Patriarcado.

\section{ADDITIONAL KEYWORDS}

Social Movements, Gender, Patriarchy.

RESUMEN. Este artículo analiza los movimientos sociales desde los enfoques constructivistas y culturales, en concreto de las tesis que defienden el papel crucial de los movimientos como creadores de nuevos marcos de interpretación de la realidad, y que investigan la influencia de esta forma de acción sobre el cambio social. Su objetivo es contribuir a una comprensión mejor de la influencia del movimiento feminista sobre los cambios que están afectando a las relaciones entre hombres y mujeres, y a sus posiciones diferenciales en la sociedad. En concreto acudimos al estudio de un caso y analizamos el papel del movimiento feminista en el reciente proceso de redefinición y deslegitimación de la violencia contra las mujeres. Este proceso ha aportado una nueva explicación de las causas de la violencia y ha significado el tránsito de este tipo de violencia del espacio privado al espacio público, y de la calificación de drama privado a su consideración como problema social.
\end{abstract}

ABSTRACT. This article adopts a constructivist and cultural focus to analyse the social movemements. In particular we follow the theses that argue that movements play a crucial role in creating new frameworks for interpreting reality. Such theses also research the influence of these frameworks on social change. Our objective is to develop a better understanding of the influence of the feminist movement on changes that are affecting the relationships between men and women, and their different positions in society. We turn to a study case and analyse the role of the feminist movement in the recent process of redefinition and rejecting of the justification of violence against women. This process has given new explanation of the causes of violence and has meant the transferal or this type of violence from the private sphere to the public sphere; also its recategorization from being a private problem to a social problem.

*Una primera versión de este artículo fue presentada como ponencia en el VII Congreso Español de Sociologia celebrado en Salamanca, en 2001. Quiero agradecer sus críticas y sugerencias a todos los participantes en el Grupo de Trabajo Movimientos Sociales y Acción Colectiva.

E-mail: anamig@udc.es

Revista Internacional de Sociología (RIS)

Tercera Época, No 35, Mayo-Agosto, 2003, pp. 127-150. 
RIS

REVISTA INTERNACIONAL DE SOCIOLOGIA

№ 35, Mayo-Agosto, 2003

ANA DE MIGUEL ÁLVAREZ

\section{APROXIMACIÓN TEÓRICA A LOS MOVIMIENTOS SOCIALES}

En las últimas décadas se han desarrollado nuevos y sugerentes enfoques teóricos sobre los movimientos sociales. A pesar de las diferencias entre los mismos, sí existe cierto consenso a la hora de considerar que los movimientos sociales presentan formas de acción y organización cuyo impacto sobre el cambio social no había sido ni comprendido ni valorado adecuadamente por los enfoques clásicos. Efectivamente, aún sin ser todos estrictamente nuevos -el movimiento feminista, por ejemplo, cuenta con más de dos siglos de existencia-, los movimientos sociales que se consolidan a partir de las nuevas condiciones sociales y de la nueva cultura política que emerge con la resaca de los años sesenta, no se ajustaban bien a los planteamientos clásicos ni a las teorías pioneras sobre el comportamiento colectivo, en las que los movimientos aparecían como producto de circunstancias excepcionales y cuya acción como masa se propagaba de forma irracional, ni a los planteamientos marxistas ortodoxos, como producto más o menos mecanicista y economicista de la lucha de clases ${ }^{1}$.

Entre los nuevos enfoques están algunos tan conocidos como el de la movilización de recursos, que prima los factores organizativos, o el de la estructura de la oportunidad política, que se centra en aspectos de la coyuntura política, y, últimamente, los que podemos agrupar bajo el rótulo de constructivistas. Entre los enfoques de orientación constructivista confluyen a su vez enfoques interaccionistas, culturales y cognitivos, es decir, aquellos en los que han primado las preguntas en torno a los procesos de interacción y elaboración de significados por los que los movimientos sociales definen su identidad, su ideología y sus reivindicaciones. Estos enfoques comenzaron a desarrollarse en Europa alrededor del concepto de identidad colectiva, en las obras de autores como Alain Touraine, y muy especialmente, del italiano Alberto Melucci. A su vez, en los Estados Unidos comenzaba a abrirse paso este enfoque a partir de la recuperación del concepto de marco, definido en su día por Goffman como el conjunto de las orientaciones mentales que permiten organizar la percepción y la interpretación (Goffman, 1974). Y, en la actualidad, la importancia de los movimientos como creadores de nuevas identidades sociales y nuevos marcos de interpretación o referencia-marcos de injusticia-, que pugnan con otros agentes sociales por hacer hegemónica su definición de la situación, no ha dejado de enriquecer el panorama teórico².

\footnotetext{
${ }^{1}$ Estos nuevos enfoques han tenido una recepción casi inmediata entre nosotros, como lo prueba la continua edición de publicaciones colectivas que se están publicando en estos años. Entre otras, Laraña, E. y J. Gusfield (eds.) (1994); Ibarra, P. y B. Tejerina (eds.) (1998); McAdam, D., J. McCarthy, M.N. Zald (eds.) (1999); Robles Morales, J.M (ed.) (2002); Funes, M.J. y R. Adell (eds.) (2003).
} 
Para los enfoques constructivistas, la identidad, la ideología y las reivindicaciones objetivas de los movimientos no se consideran como algo dado o como algo obvio y evidente en sí mismo. Muy al contrario, se considera que el proceso por el que un colectivo social llega a definir como injusta y como objeto de cambio social a una situación dada, generalmente legitimada por la tradición cultural y la costumbre, es una de las contribuciones más importantes de los movimientos al cambio social. De acuerdo con esta lógica, la visión que nos ofrecen los enfoques de la movilización de recursos de los movimientos como centros de agitación, protesta y presión no es la única adecuada. Los enfoques constructivistas presentan una imagen de las redes de los movimientos y de su acción interna y externa más cercana a los laboratorios de innovación cultural de los que hablara Melucci. En estos laboratorios fermenta lentamente la creación de nuevos marcos de referencia, de nuevos significados para interpretar los hechos. Tal y como ha escrito Gusfield, la sola existencia de un movimiento es ya un principio para situar acontecimientos en un marco; éste presenta un aspecto de la vida social que ya está sometido a discusión pública, aunque anteriormente se hubiera aceptado como la norma: "donde la elección y la disputa estaban ausentes, están ahora presentes las alternativas" (Gusfield, 1994). Esta visión, que enfatiza la relevancia de la teoría o praxis cognitiva y el protagonismo de los movimientos en los cambios culturales y de mentalidad, nos parece especialmente explicativa para valorar los profundos cambios que el feminismo está introduciendo en unas formas de interacción y organización social entre los sexos que, según parece, pueden retrotraerse hasta la noche de los tiempos.

Desde nuestra perspectiva, los enfoques que priman elementos de la cultura interna del movimiento no se oponen, sino que se complementan, con los enfoques de la movilización de recursos y del proceso político, caracterizados por medir y cuantificar la influencia de los movimientos según la organización de sus recursos, su presencia combativa en las calles y su influencia directa en los cambios políticos e institucionales. Como han señalado distintos investigadores, si la pluralidad teórica es siempre positiva y enriquecedora, aún más habrá de serlo en casos de efervescencia y renovación teórica, como indudablemente ocurre en la actualidad en el área de los movimientos sociales. Sin embargo, sí es cierto que el uso exclusivo de estos últimos enfoques ha podido llevar a caer en cierta miopía de lo visible, utilizando la afortunada expresión de Melucci, miopía desde la que se postula recurrentemente la decadencia de los movimientos sociales, bien por el hecho de que ya no celebren manifestaciones tan multitudinarias como antes, bien

\footnotetext{
${ }^{2}$ El análisis de los movimientos como creadores de marcos no implica necesariamente una visión culturalista de los mismos, es decir, no está en contradicción con mantener que la causa de que existan movimientos sociales radica en factores y conflictos estructurales.
} 
RIS

REVISTA INTERNACIONAL DE SOCIOLOCIA

№ 35, Mayo-Agosto, 2003

ANA DE MIGUEL ÁLVAREZ

porque los partidos políticos y las instituciones hayan hechos suyas parte de sus reivindicaciones. Este es, a menudo, el trato que se ha dispensado al movimiento feminista, del que buena parte de la posmodernidad ha coincidido en certificar una y otra vez su "muerte" - una veces por ineficaz, otras por innecesario- y el acceso a una bienaventurada era de postfeminismo. Frente a esta visión, la aplicación del enfoque constructivista nos permite aventurar la hipótesis de que las plurales y activas redes del movimiento no actúan sólo ni principalmente cuando movilizan sus recursos de cara a la opinión pública, sino cuando permanecen sumergidas elaborando la visión feminista de la realidad, con lo que aparecen como un factor decisivo en la paulatina configuración del feminismo como un sentido común alternativo (Sabucedo et al., 1998).

Como punto de partida de este trabajo tomamos la definición de los movimientos propuesta por Laraña, una de las más recientes y comprensivas, y que, desde una posición constructivista, enfatiza los elementos culturales y cognitivos de los movimientos. Según esta definición, los movimientos son una forma de acción colectiva "1) que apela a la solidaridad para promover o impedir cambios sociales; 2) cuya existencia es en sí misma una forma de percibir la realidad, ya que vuelve controvertido un aspecto de ésta que antes era aceptado como normativo; 3 ) que implica una ruptura de los límites del sistema de normas y relaciones sociales en el que se desarrolla su acción; 4) que tiene capacidad para producir nuevas normas y legitimaciones en la sociedad" (1999: 126-7). De acuerdo con esta definición, este trabajo trata sobre el papel central de la teoría, el conocimiento y la construcción de nuevos marcos de interpretación en el movimiento feminista. En la primera parte se defiende la tesis de que la redefinición de la realidad o praxis cognitiva, es decir, la subversión de los códigos culturales dominantes es, junto con las ya más conocidas y estudiadas políticas reivindicativas y de igualdad, una de sus prácticas fundamentales. Asimismo, y atendiendo a las relaciones entre la producción social del significado y el conocimiento académico y profesional, se aborda la reinterpretación del conocimiento académico que se ha producido desde los denominados Women's Studies que hoy se denominan cada vez más estudios de género. En la segunda parte, y con el fin de ejemplificar estas tesis, se expone el caso de la violencia contra las mujeres. Mantenemos que las recientes campañas y respuestas sociales, políticas y legales son, en realidad, el producto final, público $\mathrm{y}$ visible de un largo y complejo proceso de redefinición de la violencia contra las mujeres, que ha pasado de calificarse como un drama personal, a conceptualizarse como un problema social. Este proceso, que comenzó en el ámbito del movimiento feminista en los años sesenta, se ha desarrollado en sociedades formalmente igualitarias y con políticas públicas de igualdad, en las que las mujeres han logrado mayor protagonismo en todas los niveles del espacio público. 


\section{LA PERSPECTIVA FEMINISTA Y LOS ESTUDIOS DE GÉNERO}

\section{La teoría feminista}

La teoría feminista es, ante todo, una teoría crítica de la sociedad. En palabras de Celia Amorós, una teoría que irracionaliza la visión establecida de la realidad. Amorós nos recuerda la raíz etimológica de teoría, que en griego significa ver, para subrayar el que es el fin de toda teoría: posibilitar una nueva visión, una nueva interpretación de la realidad, su resignificación (Amorós, 1998). La teoría, pues, nos permite ver cosas que sin ella no vemos, por lo que, en este sentido, el acceso al feminismo supone la adquisición de un nuevo marco de referencia, "unas gafas" que muestran a menudo una realidad ciertamente distinta de la que percibe la mayor parte de la gente ${ }^{3}$. Efectivamente, una de las cuestiones centrales que ha tenido y tiene que afrontar el movimiento feminista es el hecho claro de que muchas mujeres no aceptan la visión feminista de la realidad. Tal y como lo enunciara Mary Wollstonecraft hace ya más de dos siglos, el hecho de que las mujeres parecieran dedicarse más a sacar brillo a sus cadenas que a tratar de sacudírselas ${ }^{4}$. En esta cuestión el feminismo coincide con otros movimientos sociales, ya que, como han puesto de relieve los nuevos enfoques, los movimientos no se explican sólo como respuestas colectivas a conflictos manifiestos o desigualdades estructurales, sino que buena parte de su sentido y acción se dirige a mostrar, a hacer explícito o visible ese conflicto para la opinión pública ${ }^{5}$. Así, por ejemplo, el movimiento ecologista trata de problematizar o redefinir situaciones que, como la destrucción de los recursos naturales o el maltrato a los animales, continúan definiéndose como naturales, inevitables o consustanciales al rango superior y al progreso de la especie humana. En este sentido preciso, todos los movimientos suponen la subversión de los códigos culturales dominantes. Pero, tal vez, la peculiaridad del feminismo resida en lo que ya señalara Kate Millett: el feminismo desafia el orden social y el código cultural más ancestral, universal y arraigado de los existentes, en sus diversas manifestaciones (Millett, 1995).

\footnotetext{
${ }^{3} \mathrm{El}$ enfoque de los marcos de referencia se inspira en la obra de Goffman y ha sido desarrollado entre otros por Gamson, Snow y Hunt. Cfr. A. Rivas (1998).

${ }^{4}$ Mary Wollstonecraft fue una de las pioneras del feminismo de raíz ilustrada. Su obra Vindicación de los derechos de la mujer, publicada en 1792, surge del asombro y la indignación por la exclusión de las mujeres de la ciudadanía, tanto en las obras de sus admirados filósofos ilustrados como en la práctica de la Revolución Francesa.

${ }^{5}$ Este imperativo de persuadir, de convencer a la opinión pública de la legitimidad de marcos alternativos de interpretación, explica el interés que han suscitado los nuevos enfoques culturales dentro del área de los movimientos sociales, lo que no implica subestimar los aspectos estructurales de los conflictos, por lo menos no en nuestro caso.
} 
En este caso las investigaciones más recientes parecen avalar las hipótesis de las primeras teóricas, a la fuerza más intuitivas (y creativas). Según éstas, la ideología patriarcal está tan firmemente interiorizada, sus modos de socialización son tan perfectos que la fuerte coacción estructural en que se desarrolla la vida de las mujeres presenta para buena parte de ellas la imagen misma del comportamiento libremente deseado y elegido ${ }^{6}$. Estas razones explican la crucial importancia de la teoría dentro del movimiento feminista, o dicho de otra manera, la crucial importancia de que las mujeres lleguen a deslegitimar "dentro y fuera" de ellas mismas un sistema que se ha levantado sobre el axioma de su inferioridad y su subordinación a los varones. La teoría feminista tiene entre sus fines conceptualizar adecuadamente como conflictos, y producto de unas relaciones de poder determinadas, hechos y relaciones que se consideran normales o naturales, en todo caso, inmutables. Aquellos de los que se suele afirmar que "siempre ha sido así y siempre lo será", en expresiones tales como "la prostitución es el oficio más viejo del mundo", o "los hombres siempre serán hombres y eso no hay quien lo cambie", en referencia a las causas de la violencia contra las mujeres. La teoría feminista indaga en las fuentes religiosas, filosóficas, científicas, históricas, antropológicas, artísticas y también en el llamado sentido común, para desarticular las falsedades, prejuicios y contradicciones que legitiman la dominación sexual. Este cuestionamiento de la realidad patriarcal puede analizarse como una sucesión de pasos o momentos teóricos y prácticos, colectivos e individuales. En un primer momento, hay que definir una situación como problemática, injusta e ilegítima; en un segundo momento, habría que encontrar las causas de esta situación, sean culturales, económicas, etc., o establecer lo que se ha denominado la atribución de la responsabilidad. En un tercer momento, el feminismo ha de ser capaz de articular propuestas alternativas: no sólo hay que definir una situación como injusta, hay que difundir también la conciencia de que es posible cambiar la sociedad $y$, en última instancia, universalizar esta conciencia, desarrollar la imaginación feminista lo suficiente como para hacer irrenunciable esa sociedad nueva, para mostrar cómo todos los seres humanos se beneficiarán del cambio. Respecto a esta última afirmación podemos añadir que mantener que todos los seres humanos se benefician de un cambio no está en contradicción con que algunos colectivos pierdan privilegios, porque, obviamente, los varones pierden y perderán numerosos privilegios ilegítimos según avance el feminismo. El fin de este proceso -si es que tiene fin, porque, como ha señalado Amorós, los pactos patriarcales son metaestables (Amorós,1998), es decir, susceptibles de transformar continuamente sus formas de dominación, tan distintas las de hace dos siglos de las de ahora, tan

\footnotetext{
${ }^{6}$ Véanse, por ejemplo, los sugerentes trabajos empíricos de Davies sobre la transmisión del género en la etapa preescolar: B. Davies (1995).
} 
distintas en las diferentes culturas, pero siempre tan eficaces ${ }^{7}$ - tiene como resultado lo que se ha denominado la liberación cognitiva (McAdam, 1982), la puesta en tela de juicio de principios, valores y actitudes aprendidos e interiorizados desde la infancia y, por supuesto, el paso a la acción, tanto individual como colectiva.

Desde este doble nivel micro-macro, la práctica del movimiento feminista se ha desarrollado conjugando ambos tipos de acción, individual y colectiva, con el fin de socavar la doble reproducción del sistema patriarcal, dentro y fuera de las personas, en el espacio privado y en el público, para romper la dinámica de refuerzo mutuo entre las prácticas de la vida cotidiana y las macroestructuras económicas, políticas e ideológicas. Ha supuesto, además, la constitución de una identidad colectiva feminista, un Nosotras articulado en función de los intereses específicos de las mujeres como mujeres, capaz de abstraer las profundas diferencias que por fuerza ha de tener un sujeto colectivo que afecta a la mitad de la humanidad (Valcárcel, 91). Nos referimos tanto a diferencias socioeconómicas como ideológicas y culturales ${ }^{8}$. Pues bien, tal y como hemos desarrollado en otro lugar, la articulación de esta identidad feminista se ha realizado históricamente desde dos tipos de prácticas fundamentales que son dos formas de intervención sobre la realidad: la deslegitimación del entramado conceptual patriarcal o la redefinición de la realidad y el activismo en torno al conjunto de reivindicaciones que de ahí se deriva (de Miguel, 2002); donde las reivindicaciones, como ya señalara Mueller, no pueden entenderse como algo dado, lógico o natural (Mueller, 1994). $\mathrm{Al}$ contrario, las reivindicaciones de las mujeres son el resultado visible y final de intensos procesos colectivos de elaboración de nuevos marcos de injusticia.

\section{Hacia un nuevo paradigma: rupturas conceptuales}

Los célebres años sesenta, años en que resurge con fuerza el movimiento feminista, trajeron un legado crucial a las nuevas generaciones de mujeres. Por un lado, sacudieron con una fuerza inusual las viejas tradiciones y mentalidades: las mujeres dijeron adiós a la mística de la feminidad y emprendieron lo que Betty Friedan denominaría el viaje apasionado hacia su liberación. Por el lado teórico, la publicación de dos clásicos del feminismo, como son Política sexual de

\footnotetext{
${ }^{7}$ Alicia Puleo ha distinguido entre patriarcados basados en la coacción y patriarcados basados en el consentimiento. En los primeros, el propio sistema ideológico y legal establece la inferioridad de las mujeres y consigna prohibiciones y castigos explícitos. En los segundos, la socialización diferencial encubierta, las prácticas discriminatorias arraigadas en el mercado laboral y la difusión de poderosos mitos patriarcales a través de los medios de comunicación propician la aceptación 0 la resignación ante la desigualdad (Puleo, 1995).

${ }^{8}$ Lógicamente la situación específica de cada mujer o colectivo de mujeres se cruza también con otras identidades, como pueden ser la de clase, edad, opción sexual y cultura.
} 


\section{RIS}

Kate Millett y La dialéctica de la sexualidad de Sulamith Firestone, contribuyó decisivamente a desbrozar el camino abierto en su día por la obra pionera de Simone de Beauvoir, El segundo sexo, y por el que habría de discurrir la teoría feminista posterior. Los términos de un conflicto que ya no podía subsumirse en el de clases y los nuevos temas de investigación habían quedado planteados; quedaba por ver cuántas, pasados los momentos de mayor euforia y presencia pública del movimiento, recogerían el guante. La verdad es que resultaron ser suficientes y la teoría feminista comenzó su lenta pero imparable transformación del conocimiento (Harding, 1991). Tal y como se ha dicho, sólo cuando las mujeres se constituyeron en sujetos de investigación es cuando entraron a formar parte del cuadro, cuando se constituyeron, también, en objetos de investigación. Para Lengermann y Niebrugge-Brantley, el surgimiento de los estudios de género arranca de un interrogante engañosamente sencillo: “ $¿ Q u e ́$ hay de las mujeres? En otras palabras, ¿dónde están las mujeres en la situación que se está investigando? Si no están presentes, ¿por qué no lo están? Y, si lo están, ¿qué es lo que hacen exactamente?, ¿cómo experimentan la situación?, ¿cómo contribuyen a ella?, ¿qué significa para ellas?" (Lengermann, 1993).

El reto de las nuevas teorías y las investigaciones de género será crear conceptos capaces de captar y hacer visible la especificidad de la situación de las mujeres en todos y cada uno de los momentos y lugares de la vida social, política, económica, cultural, artística, deportiva, etc, de identificar los mecanismos sociales que permiten y propician la desigualdad sexual. En este sentido, los estudios feministas y de género responden a la caracterización que ha realizado John Rex del enfoque teórico del conflicto: su tarea no sólo reside en analizar el desarrollo de los conflictos reconocidos como tales, sino, muy especialmente, en generar conceptos y teorías susceptibles de poner de manifiesto los conflictos, la coacción, bajo la apariencia de consenso y libertad (Rex, 1985). Efectivamente, el conflicto de género ha sido y continúa siendo uno de los más difíciles de aprehender por las ciencias sociales. En primer lugar, porque en una sociedad como la nuestra, en la que las desigualdades legales prácticamente han desaparecido - las mujeres no sólo pueden ir a la universidad y votar, sino que siempre hay alguna en cada gobierno-, no resulta una tarea fácil hablar de desigualdad, de opresión (¿dónde está?). Las elecciones de las mujeres, fundamentalmente distintas cuando no opuestas a las de los varones, presentan la apariencia de la elección. ¿No sería, pues, realmente su "naturaleza" lo que determinaba sus elecciones? Tal y como dijera Betty Friedan, el problema de las mujeres es "el problema que no tiene nombre" y el fin de la teoría feminista es, ante todo, el de establecer su adecuada conceptualización. En segundo lugar, también hay que destacar el problema de que los indicadores habituales para estudiar otros conflictos también contribuyen a invisibilizarlo. Por ejemplo, en el conflicto de géneros no podemos contabilizar el número de huelgas, o de horas perdidas de trabajo asalariado, tampoco el número de trabajadores implicados. $Y$ tampoco suelen las mujeres emplear la violencia 
como estrategia de apoyo a sus reivindicaciones, más bien son víctimas de ella. No retienen directivos, no hacen piquetes de huelga, no agreden a las fuerzas del orden público. Así, frente al alto grado de visibilidad que adquieren otros conflictos, amplificados en los medios de comunicación, parece que no existe éste. De ahí la necesidad y la importancia de conceptualizar y enmarcar el problema para hacer visible esta realidad.

\section{Dos conceptos para un nuevo marco de análisis}

Los conceptos de género y patriarcado han llegado a convertirse en dos conceptos fundamentales, tanto por su elaborada capacidad de síntesis para hacer referencia a una realidad compleja, como por su generalizada aceptación por parte de la comunidad feminista y académica. Con ellos la teoría feminista ha logrado introducir su visión de la realidad en la visión hegemónica del conocimiento y la mayor parte de las disciplinas humanísticas y sociales. Ha logrado que la "ciencia normal" abandone el farragoso terreno de la "naturaleza" para explicar - u ocultar- la situación social de las mujeres, para contestar la cuestión de "¿qué pasa con las mujeres?". Se entiende por género o sexo-género la construcción social de la diferencia sexual entre varones y mujeres. El concepto de género, en principio, ni cuestiona ni considera especialmente relevantes las diferencias biológicas entre los dos sexos. Lo que sí niega es la traducción causal de las diferencias anatómicas en "naturalezas sociales" o caracteres distintos. Lo femenino y lo masculino son categorías sociales y la perspectiva del género invita a investigar cómo se construyen y cómo operan organizacionalmente estas definiciones. Además, se considera que el género es un principio organizativo fundamental de la vida social y de la conciencia humana. No hay esfera o nivel de la vida humana y social que no sea susceptible de un análisis de género (Alberdi, 1999).

El concepto de patriarcado - algunas autoras prefieren utilizar el de sistema de estratificación sexual o sistema de sexo-género-muestra que la construcción social de las diferencias fisiológicas está relacionada con la jerarquización y las relaciones de dominación entre los géneros, jerarquización que es la característica principal de una sociedad patriarcal. En este sentido, puede afirmarse que es el patriarcado el que crea los géneros. Tal y como ha argumentado Amorós, una sociedad igualitaria no produciría la marca de género, signo de la pertenencia a un grupo social con determinadas características y funciones (Amorós, 1998). Es decir, igual que hay clases porque hay relaciones de dominación entre ellas, hay géneros porque median relaciones jerárquicas entre los mismos. La utilización del concepto de patriarcado siempre ha suscitado menos consenso que el de género. Inicialmente fue utilizado por el feminismo radical para marcar la especificidad de la dominación de las mujeres frente a otros tipos de dominación, así como para señalar a los varones -y no ya al capitalismo o al "Sistema" - como los beneficiarios de la misma. Como señalara Millett en su obra Politica Sexual, no 
se conoce sociedad alguna en la que las mujeres tengan más poder que los varones, de ahí que pueda afirmarse que es el sistema de dominación más universal y longevo existente (Millett, 1995).

En la actualidad, las críticas al concepto de patriarcado se centran en apuntar a su carácter ahistórico y a su elevado nivel de abstracción, que diluye las situaciones reales y diversas de las mujeres. También puede mencionarse otro tipo de críticas de índole bien diferente: las que mantienen que el patriarcado ya ha desaparecido. Sin embargo, autoras como la politóloga nórdica Jónasdóttir (1993) han señalado que este concepto es adecuado en el nivel más general de la teoría, nivel en que es comparable al concepto de "sociedad de clases". Por consiguiente, no proporciona, ni pretende hacerlo, una opción teórica determinada sobre cómo se estructura y reproduce la desigualdad en las situaciones socio-históricas concretas. Y, en consecuencia, tampoco anula las cambiantes y diversas experiencias históricas de las mujeres y los continuos reajustes de las relaciones entre los sexos. La bondad del concepto reside, en palabras de la autora de El Contrato Sexual, en su capacidad para singularizar la situación de las mujeres como tales (Pateman, 1995). Situación que, por supuesto, interactúa con el resto de las posiciones sociales como la clase, la raza o la opción sexual. En definitiva, el concepto de patriarcado remite a una situación de conflicto por la distinta posición de poder que ocupan mujeres y varones en este sistema de estratificación o dominación, mientras que el de género hace referencia a que esta situación es susceptible de cambio y transformación. Ambos constituyen una sólida base conceptual desde la que rivalizan las diferentes teorías que pugnan por explicar en qué consiste fundamentalmente y cómo se reproduce la dominación sexual.

\section{Las prácticas de redefinición en torno a la dicotomía público-privado}

A pesar de la pluralidad teórica - y de los enconados debates teóricos internos-que caracteriza la perspectiva feminista, numerosas investigaciones han coincidido en señalar la estrecha conexión entre el sistema patriarcal y la organización social de dos espacios distintos y jerarquizados donde se desarrolla la acción social. Son el espacio público y el espacio privado. A partir de ellos, es posible distinguir entre las teorías que han investigado la situación de las mujeres en la esfera pública-mercado de trabajo, educación, ideología patriarcal, etc.- y las teorías que han dirigido su atención a la esfera privada y, en general, a las relaciones entre varones y mujeres en el marco de la familia, las relaciones amorosas y la sexualidad (Salztman, 1992). La persistencia de la desigualdad respecto a los varones en el acceso a la esfera pública, fuente de recursos y poder, ha dado lugar a nuevas reivindicaciones destinadas a romper ese techo de cristal, no por transparente menos eficaz. En este orden se inscriben demandas como la de una democracia paritaria, en continuidad con las políticas que sostienen que el camino hacia la igualdad sexual es el camino hacia la progresiva inclusión de las mujeres en el espacio público'. Sin embargo, como 
suele decirse, un enfoque teórico es como la luz: ilumina una parte de la realidad, pero, inevitablemente, deja en la sombra otra parte. De este modo, el enfoque anterior ilumina la exclusión de las mujeres del espacio público, pero deja en la sombra las interacciones de varones y mujeres en el espacio privado. Y, de alguna manera, pareciera proyectar la imagen estereotipada de una mujer ama de casa que pasa sus horas mano sobre mano. Es decir, la idea de que las mujeres no realizan ningún trabajo productivo dentro del hogar.

El enfoque de la esfera privada dejará de lado, inicialmente, las investigaciones en los niveles macro para centrarse en el nivel microsocial. Tomando prestado el revelador título de una de las obras clásicas de María Angeles Durán (1988), la pregunta que guiará estas teorías e investigaciones será: ¿qué pasa "de puertas adentro"?, ¿qué hacen las mujeres?, ¿qué significado tiene para ellas? Frente al estereotipo de que las mujeres no trabajan, fuertemente reforzado por definiciones sociales del tipo "mi mujer no trabaja" o "mi madre no trabaja", las nuevas investigaciones han puesto de relieve la cantidad de horas de trabajo que pueden subyacer al estereotipo de "sus tareas". Este trabajo, que no sólo comporta cargas físicas, sino psicológicas y morales -el cuidado de niños, mayores y enfermos-, es especialmente susceptible de extenderse durante las veinticuatro horas del día, generalmente sin vacaciones. De ahí que, frente a la definición de "no trabajo" para las tareas que realizan las mujeres en el ámbito doméstico, éstas se hayan redefinido como "la jornada interminable". El análisis de la esfera privada también ha arrojado luz sobre la denominada "doble jornada laboral" que padecen las mujeres que se han incorporado al trabajo asalariado. La doble jornada hace referencia a la falta de reciprocidad en el reparto del trabajo doméstico. La constatación de esta fuerte desigualdad ha propiciado una redefinición de las políticas de igualdad. En primer lugar, la situación de las mujeres ya no se define fundamentalmente como una situación de desigualdad en el espacio público. No se niega la exclusión, pero se afirma que la mera inclusión no resuelve el conflicto ni transforma necesariamente las relaciones entre los géneros. Según este análisis, mientras la desigualdad en la esfera privada continúe reproduciéndose, la igualdad en la esfera pública es una vana quimera. Por otro lado, dirigir la luz hacia la esfera privada y las interacciones cara a cara - desde el preclaro lema feminista "lo personal es político"- ha llevado a la esfera pública el análisis de relaciones tan supuestamente privadas como la sexualidad, la heterosexualidad, la maternidad y el amor. Asimismo, frente al "hogar, dulce hogar" se descubrirá "la cara oculta de la familia": la familia como el

\footnotetext{
${ }^{9}$ En la actualidad la democracia paritaria se ha fundamentado de dos formas opuestas. Desde el feminismo de la igualdad supone una medida más de acción positiva, cuyo fin es contrarrestar las elevadas "cuotas" de representación masculina en la vida política. Para el feminismo de la diferencia supone el reconocimiento político de la diferencia ontológica, es decir, irreductible, entre los sexos.
} 
RIS

REVISTA INTERNACIONAL DE SOCIOLOGIA

№ 35, Mayo-Agosto, 2003

ANA DE MIGUEL ÁLVAREZ

ámbito de la alienación, cuando no de los malos tratos y el abuso sexual. El trabajo pionero de Susan Brownmiller (1975), que analiza la violación como una estrategia de dominación por medio del temor que infunde a todas las mujeres, puso las bases del proceso de redefinición o atribución de nuevos significados a la violencia contra las mujeres, un largo y combativo proceso sostenido por el movimiento feminista y que ha conducido a la actual aceptación social de la redefinición del fenómeno como terrorismo doméstico. Y también a solicitar la intervención pública o del Estado, vía derecho penal y asistencia social, en áreas de la vida tradicionalmente consideradas privadas o personales. Estos ejemplos anteriores nos permiten seguir el camino que ha llevado al feminismo a plantear como uno de sus fines la redefinición -no la abolición- de la división tradicional entre lo público y lo privado.

En este sentido, innovadores trabajos de teoría feminista han coincidido en concluir, desde distintos referentes filosóficos, históricos y sociológicos," que la exclusión de las mujeres de la categoría de sujetos y ciudadanas en la modernidad no puede considerarse un "mero accidente o una aberración". Muy al contrario, se apunta la tesis de que en la teoría social y política modernas, la constitución de lo público se habría realizado gracias a la exclusión-necesaria exclusión, por tanto-de aspectos fundamentales de la vida humana, y estos, a su vez, gracias a la exclusión -necesaria también - de las mujeres. Desde esta perspectiva, e intentando obviar cualquier zona de sombra, un tema clave en las investigaciones actuales sobre los géneros es el de la articulación o las relaciones entre lo público y lo privado, donde lo privado aparece como la condición de posibilidad de la esfera pública (Castells, 1998). Desde esta articulación se explican las enormes dificultades y obstáculos que experimentan las mujeres para participar en igualdad de condiciones en un espacio que se ha configurado - simbólica y materialmente-, no ya a partir de su exclusión, sino de su participación a tiempo completo en las tareas de la reproducción social. La capacidad de dar cuenta de estos realineamientos y transformaciones ha supuesto la necesidad de rupturas conceptuales y alternativas teóricas para deshacer la intrincada madeja del androcentrismo en las ciencias sociales. Por ejemplo, respecto al crucial tema del trabajo: "Estas alternativas plantearán, en lo fundamental, conceder a la organización social de la reproducción humana la misma importancia conceptual que a la organización de la producción asalariada" (Borderías, Carrasco y Alemany, 1994). Planteamiento en el que subyace la idea de que las mujeres no necesitan reivindicar para sí mismas lo que ya tienen en demasía - tareas domésticas, ética del cuidado, etc.- - sino conseguir dotar de universalidad lo que el sistema patriarcal ha considerado y quiere seguir considerando como valores y actividades femeninas.

Por su parte, estas rupturas conceptuales son producto y encuentran un correlato político en la continua y creativa práctica militante de las redes del movimiento feminista, y en otras acciones políticas más visibles e institucionales difundidas por los medios de comunicación, como las Conferencias Mundiales sobre las mujeres y las Declaraciones de Derechos de las mujeres, como la Declaración de Atenas de 
1992 y la Declaración de Pekin de 1995. Estas declaraciones han sido el resultado de los pactos entre mujeres de diferentes países, culturas e ideologías, pero que han llegado a constituirse en un sujeto colectivo que ha expresado su voluntad de firmar un nuevo Contrato Social; es decir, de poner fin a la adjudicación de espacios, identidades y funciones sociales según el sexo, que configuran la base de las democracias actuales. Acabar, y no únicamente en el orden simbólico, con la identificación patriarcal de lo masculino con lo humano y lo femenino con lo específico de las mujeres. Las mujeres, que han luchado durante siglos por la autonomía económica y política, han llegado a postular también la necesidad de autonomía discursiva, interpretativa y comunicativa (Fraser, 1997).

\section{REDEFINICIÓN DE LA VIOLENCIA CONTRA LAS MUJERES}

En este apartado vamos a presentar el tema de la violencia de género como uno de los casos en donde se puede apreciar de forma más clara el papel central de la teoría o la elaboración de nuevos marcos de referencia para conferir nuevos significados a hechos viejos, siendo para ello de vital importancia, tanto las elaboraciones internas desde dentro del movimiento feminista - autonomía interpretativa -, como la posterior difusión de dicha interpretación de la realidad a toda la sociedad, creando un nuevo significado compartido o un sentido común alternativo - autonomía comunicativa. Partimos pues de que la violencia contra las mujeres no es, ni mucho menos, una realidad nueva. Lo realmente nuevo es el significado social que se le ha llegado a atribuir en la actualidad. El siglo diecinueve, por ejemplo, abunda en testimonios sobre la gravedad y la generalización de esta violencia. Dos autores, tan lejanos entre sí respecto a otros problemas sociales como lo son John Stuart Mill y Friedrich Engels, coincidieron en denunciar la brutalidad y la práctica impunidad legal de la misma. Dos socialistas utópicas tan relevantes como Flora Tristán y Anna Wheeler soportaron continuos malos tratos que, en el caso de la francesa, llegaron al intento de asesinato. Sin embargo, como han señalado diversas autoras, sólo recientemente, y en relación con la mejora generalizada del status de las mujeres, la violencia doméstica ha dejado de considerarse un "problema personal" de las mujeres afectadas para considerarse un "problema social" (Ferrer y Bosch, 2000, 2002).

Hasta fechas no tan lejanas las explicación hegemónica de los malos tratos a las mujeres ha sido de corte biologista, psicologista e individualista. Según esta explicación, tan académica como "popular", la violencia masculina, ciertas dosis de violencia masculina, se ha considerado algo natural, relacionado con la supervivencia de la especie y ya observable en los niños varones, caracterizados por la actividad y la competitividad frente a la pasividad y dulzura de las niñas. En consecuencia, sólo los casos extremos, es decir, las actitudes especialmente violentas y sanguinarias, se llegaban a interpretar como producto de diversas patologías individuales (Alberdi, 2002). Otra explicación recurrente ha consistido en confundir las causas 
RIS

REVISTA INTERNACIONAL DE SOCIOLOCIA

№ 35, Mayo-Agosto, 2003

ANA DE MIGUEL ÁLVAREZ

de la violencia con factores que frecuentemente se presentan asociados a la misma, como pueden ser el alcoholismo o la insumisión de las mujeres (abandono del hogar, separación). Tal y como se puede apreciar en los resultados de la encuesta del Eurobarómetro - realizada a petición de la Comisión Europea en el marco de la campaña Europea contra la violencia doméstica (Tabla 1) -, el alcoholismo continua encabezando aún las atribuciones causales de los malos tratos por parte de la opinión pública. Asimismo, los resultados de un reciente trabajo en el que se han investigado las atribuciones causales por parte de las propias maltratadas sitúan el consumo de drogas y alcohol como causa sea más frecuente de la primera vez que tuvo lugar el maltrato. Sin embargo, resulta significativo que al ser preguntadas por las causas a las que atribuyen el resto de los episodios de maltrato, el mayor porcentaje sea para "hechos triviales", a saber, que la cena esté fría, que los niños no estén bañados, etc. (Matud et al., 2003). Resulta significativo porque, seguramente, casi nadie acceptaría estos hechos como casusas reales del maltrato.

En la actualidad este tipo de explicaciones ha sido duramente cuestionada desde la perspectiva feminista y los estudios de género. En esta línea hay que recordar que las teóricas de la llamada segunda ola del movimiento feminista (años sesenta) ya atribuyeron a causas sociales el recurso a la violencia e identificaron el importante papel que cumple la coacción, aún en la forma de mera amenaza y posibilidad latente, en la aceptación del sistema patriarcal. En su obra Política sexual, Kate

Tabla 1.

¿Cuáles son las causas de la violencia que identifican los europeos?

\begin{tabular}{ll}
\hline Causas & $\%$ respuestas \\
\hline Alcoholismo & 96,2 \\
Adicción a las drogas & 93,7 \\
Desempleo & 78,5 \\
Pobreza y exclusión & 74,6 \\
Haber sido uno mismo víctima de malos tratos & 73,2 \\
Estar genéticamente predispuesto al comportamiento violento & 64,2 \\
El modo en el que los varones ven a las mujeres & 63,9 \\
El modo en el que el poder está distribuido entre los sexos & 59,0 \\
Un bajo nivel educativo & 57,1 \\
Los medios de comunicación & 48,6 \\
Creencias religiosas & 47,0 \\
Comportamiento provocador por parte de las mujeres & 46,1 \\
\hline
\end{tabular}

Fuente: Eurobarómetro Standard 51,0. Datos técnicos: Encuesta realizada por encargo de la Comisión Europea. Universo: 16.179 entrevistas en los 15 Estados miembros. 1000 encuestas cara a cara en España. Encuesta realizada entre el 12 de Marzo y el 4 de Mayo de 1999. 
Millett escribe: "No estamos acostumbrados a asociar el patriarcado con la fuerza. Su sistema socializador es tan perfecto, la aceptación general de sus valores tan firme y su historia en la sociedad humana tan larga y universal, que apenas necesita el respaldo de la violencia". Sin embargo, continúa Millett, "al igual que otras ideologías dominantes, tales como el racismo y el colonialismo, la sociedad patriarcal ejercería un control insuficiente, e incluso ineficaz, de no contar con el apoyo de la fuerza, que no sólo constituye una medida de emergencia, sino también un instrumento de intimidación constante" (Millett, 1995).

En general, las explicaciones sociológicas y feministas sobre el uso de la violencia contra las mujeres han resaltado dos factores causales (Maquieira y Sánchez, 1990). En primer lugar, el proceso de socialización diferencial de los sexos. Independientemente de cómo sean las hormonas masculinas hoy existe amplia documentación sobre cómo en la socialización de los varones se identifica lo masculino con la fuerza y la violencia. Además, basta con entrar a una juguetería: juguetes bélicos para los niños y juguetes domésticos para las niñas (Miedzian, 1991). En segundo lugar, se apunta a la persistencia de las definiciones sociales que representan las relaciones entre los géneros como relaciones de subordinación, cuando no de propiedad, en las que las mujeres deben cierta sumisión a sus maridos o compañeros. Será entonces, cuando las mujeres no respondan a las expectativas, cuando los conflictos pueden llevar al uso de la violencia como medio de restablecer la satisfacción de las expectativas sobre el comportamiento femenino. En este segundo caso, la violencia aparece como un efectivo medio de control social sobre el comportamiento de las mujeres. Desde esta perspectiva, es imprescindible citar el trabajo pionero de Susan Brownmiller sobre la violación. Esta autora define la violación como parte de un sistema de control que afecta al comportamiento cotidiano de todas las mujeres. Este trabajo fue considerado, en su día, exagerado y radical. Sin embargo, hoy, el propio Giddens, en su muy difundido manual de Sociología, ha llamado la atención sobre cómo el miedo a esta agresión conduce a las mujeres a ejercer un riguroso control sobre sus acciones y movimientos en el espacio público, y cómo, por tanto, funciona como un mecanismo eficaz para retenerlas en el espacio que siempre les asignó el patriarcado: el espacio privado.

La tesis central de la obra de Brownmiller - Against Our Will (1975)- sostiene que la violación forma parte del proceso de intimidación masculina del que son víctimas todas las mujeres, no sólo las que han sido violadas. El sentido de esta tesis se hace patente cuando consideramos los resultados de su investigación: 1) que mujeres que nunca han sido violadas muestran una ansiedad similar a las mujeres que sí lo han sido, y 2) que esta ansiedad y temor afecta al comportamiento de todas las mujeres, que, para evitar la posibilidad de ser violadas, tienen que aceptar limitar considerablemente su autonomía en el espacio público: no salir de noche ni por lugares solitarios, no volver tarde del trabajo, no abrir la puerta a desconocidos, no entrar con un varón a un ascensor, no escribir el nombre en el buzón. Estos son algunos de los consejos dados por una organización feminista 
norteamericana para minimizar los riesgos de victimización. Pero hay otro que subyace a todos ellos: es posible que una mujer realice tranquilamente todas las actividades anteriores siempre y cuando viva con o vaya acompañada de un varón. Según este análisis, el mensaje de la violencia o la violencia latente contra las mujeres está muy claro: una mujer "sola" está en peligro. Como ha señalado Amorós, en una sociedad patriarcal la mujer que no pertenece a ningún varón en particular pertenece potencialmente a todos, es la célebre "mujer pública"10. Ahora bien, el trabajo de Brownmiller tampoco da cuenta de otras formas de violencia. Una nueva ola de estudios ha arrojado otra perspectiva sobre la violencia contra las mujeres: si tenemos en cuenta los datos que indican que la violación por parte de extraños se produce sólo en la mitad de los casos denunciados, tampoco parece que estar acompañadas sea la solución básica para las mujeres. Y, de hecho, en estos momentos parece probarse que una parte significativa del total de las mujeres asesinadas lo son a manos de maridos, ex maridos, novios y ex novios, no precisamente desconocidos. Pero, estos hechos aparentemente contradictorios -la victimización de las mujeres tiene lugar tanto cuando están "solas" en la esfera pública como cuando están "acompañadas" o casadas en la esfera privada- pueden no serlo tanto. Como han puesto de relieve Alberdi y Matas en su reciente y exhaustivo Informe sobre los malos tratos a mujeres en España, la mayor parte de las mujeres asesinadas están en proceso de separación, es decir, han manifestado la firme decisión de volver a estar "solas" (Alberdi y Matas, 2002).

\section{La irrupción de las víctimas y el debate en torno al Derecho penal}

El avance hacia sociedades más igualitarias junto con la progresiva aceptación de los marcos de interpretación desarrollados por el movimiento feminista explican, en buena medida, la deslegitimación de la violencia contra las mujeres y su reconceptualización como un problema social. Sin embargo, este proceso, en lo referente a la criminalización y condena pública de los maltratadores, y visibilización, atención y apoyo a las víctimas, también puede encuadrarse en un proceso más general de redefinición de la violencia, la desviación y el papel social del Derecho penal. Comenzaremos haciendo algo de historia. En los años sesenta había surgido, de la mano del interaccionismo simbólico, un nuevo y radical enfoque de la desviación:

\footnotetext{
${ }^{10}$ Como es sabido, la expresión "mujer pública" ha sido sinónimo de prostituta. Aunque puede suponerse que esta acepción está en declive, la reciente edición del Diccionario de Uso del Español (1998) de María Moliner en su entrada "Mujer" incluye la definición de "mujer pública": (del punto o de la vida). Prostituta.
} 
la teoría del etiquetaje. Esta teoría supuso una inversión de la imagen positivista del desviado, que pasaba de ser el peligro social número $\mathrm{x}$, a considerarse una "creación" y una víctima del control social. Frente al enfoque correccional, se imponía una nueva actitud: escuchar, comprender sus razones, "simpatizar" con el desviado. El posterior desarrollo marxista de estos planteamientos en la obra de Taylor, Walton y Young La nueva criminología (1977) insistirá en la imagen del desviado como un rebelde político, lo sepa o no, transgresor del orden capitalista y la moral burguesa. En este contexto "descriminalizador", la irrupción de las mujeres como sujetos de conocimiento supuso un importante revulsivo a esta visión romántica y antisistema de la violencia y la desviación. Efectivamente, el análisis de género ha planteado con inusitada dureza la condición de víctimas de las mujeres en la sociedad patriarcal: ¿son acaso progresistas los malos tratos o el acoso sexual?, ¿qué significado profundamente contracultural pueden tener pintadas del tipo "si la violación es inevitable, relájate y disfruta"?, ¿qué dosis de transgresión y romanticismo - tan bien y tan machaconamente fundamentadas por reconocidos pensadores, literatos y cineastas- podemos encontrar en el ejercicio de la prostitución? Si bien las mujeres no fueron las únicas en centrarse en las olvidadas víctimas, sus análisis fueron especialmente valiosos a la hora de establecer la relación entre las víctimas y una estructura de poder determinada, en este caso, la estructura patriarcal. El descubrimiento de la relación entre la sociedad patriarcal

Tabla 2.

¿Qué fórmulas para combatir la violencia doméstica contra las mujeres consideran útiles los europeos?

\begin{tabular}{lc}
\hline Fórmulas & $\%$ respuestas \\
\hline Castigo a los agresores & 95 \\
Formar a la gente joven en el respeto mutuo & 91 \\
Una aplicación más dura de las leyes existentes & 91 \\
Un número de teléfono gratuito & 89 \\
Leyes más duras & 86 \\
Folletos informativos & 86 \\
Leyes que prevengan la discriminación sexual & 85 \\
Campañas de sensibilización & 84 \\
Tarjetas con números de contacto para urgencias & 81 \\
Formación de agentes de policía sobre los derechos de las mujeres & 78 \\
Rehabilitación de los agresores & 65 \\
\hline
\end{tabular}

Fuente: Eurobarómetro Standard 51,0. Datos técnicos: Encuesta realizada por encargo de la Comisión Europea. Universo: 16.179 entrevistas en los 15 Estados miembros. 1000 encuestas cara a cara en España. Encuesta realizada entre el 12 de Marzo y el 4 de Mayo de 1999. 
y la victimización de las mujeres supuso la aparición del debate en torno al uso del derecho penal como instrumento de cambio social progresista y feminista. De hecho, si atendemos a la opinión pública, entre las fórmulas más elegidas para combatir la violencia doméstica contra las mujeres (Tabla 2) figuran tanto el castigo a los agresores ( $95 \%$ de los encuestados), como la aplicación más dura de las leyes existentes ( $91 \%$ de los encuestados). Este renovado debate tiene entre sus protagonistas al movimiento feminista, pero puede también contextualizarse en el marco más amplio de una relativamente novedosa y sorprendente concepción del derecho penal por parte de los nuevos movimientos sociales.

La criminología crítica había etiquetado el derecho penal como un instrumento de clase al servicio del poder. Sin embargo, de esta posición podían seguirse dos posturas contrapuestas por parte de quienes impugnan dicho poder: o bien rechazar el derecho penal, o bien reivindicar una aplicación más igualitaria del mismo. Pues bien, esta segunda opción parece haber logrado, no sin fuertes tensiones, cierta hegemonía en la década de los ochenta. Así lo relata Larrauri, a quien hemos venido siguiendo en este apartado: "A partir de entonces lo que se observa con desmayo es la facilidad con que los movimientos progresistas recurren al derecho penal. Grupos de derechos humanos, de antirracistas, de ecologistas, de mujeres, de trabajadores, reclamaban la introducción de nuevos tipos penales" (Larrauri, 1991: 217). La contradicción salta a la vista. Los mismos movimientos que tradicionalmente han combatido la bondad del derecho penal y la cárcel como instrumento de resocialización y moralización públicas reclaman ahora su valor simbólico, así como "penas ejemplares". No es éste el lugar adecuado para sintetizar un debate realmente complejo, pero sí para terminar con alguna reflexión al respecto. En primer lugar, el feminismo, precisamente por ser un movimiento social, se caracteriza por una más que notable heterogeneidad de posturas y estrategias. Dentro de la gama de posturas, un extremo es el adoptado por algunos grupos feministas de la diferencia, que rechazan acudir a la ley, ley que consideran hecha "por y para varones" (Posada, 2001). Sin embargo, dejando de lado esta postura extrema, y matizando que ningún grupo confía en la mera represión vía derecho penal como única solución, sí parece existir cierto consenso sobre la necesidad de ampliar y endurecer los tipos penales y exigir una contundente y severa aplicación de la ley. Entre otras razones, por el ejemplar valor simbólico del castigo, ya que aquellas conductas que no están penadas no parecen especialmente graves. En segundo lugar, es cierto que esta actitud encierra contradicciones, ya que son bien conocidas las deficiencias del sistema penal y carcelario como agentes de rehabilitación y reinserción social, pero, en todo caso, supone la asunción de una jerarquía de valores y actuaciones donde el peligro y la protección de las víctimas se ha situado, finalmente, en el primer lugar. 


\section{CONCLUSIONES}

El éxito de las recientes y continuas movilizaciones, solicitando medidas urgentes y contundentes contra la violencia hacia las mujeres, no ha dependido solamente de la existencia de dicha violencia, ni de su supuesto aumento, sino de la forma en que el movimiento feminista ha llegado a enmarcar esta violencia y del proceso de difusión y aceptación de este nuevo marco interpretativo. El primer paso de este proceso ha sido la definición de la violencia como producto del sistema de dominación patriarcal o del sexismo, con la consiguiente ruptura de su definición tradicional como problema o desgracia personal. El segundo paso ha consistido en la aceptación y puesta en marcha de una estrategia criminalizadora del agresor y una dura crítica del tradicional olvido de las víctimas por parte del sistema penal. Con el nuevo diagnóstico de la situación y una estrategia común ante la opinión pública, el tema de la violencia llegó a los foros institucionales y se convirtiô en uno de los objetivos estratégicos de intervención de la IV Conferencia Mundial sobre las Mujeres. Pekin (1995), para, finalmente, y de la mano de algunos casos especialmente graves, alcanzar una notable y efectiva difusión en los medios de comunicación.

El éxito de estas movilizaciones, entendidas como la culminación de un largo proceso puesto en marcha por las redes sumergidas del movimiento feminista, nos lleva a prestar una renovada atención a la dimensión cultural y cognitiva de redefinición de la realidad por parte de los movimientos sociales. El movimiento feminista tiene como objetivo explícito poner fin a una de las desigualdades más universales y longevas. Sin embargo, como señalábamos en la primera parte, uno de los problemas del movimiento ha sido el de hacer visible e injusta esta desigualdad para la mayor parte de la opinión pública. Este problema continúa teniendo más vigencia, si cabe, en sociedades que, como la nuestra y el resto de las occidentales, han puesto fin a la práctica totalidad de las desigualdades formales. Y la tarea no es fácil porque también se ve dificultada por la fuerte y continua reacción ideológica en contra del feminismo. Faludi ha documentado los comienzos de esta reacción en la década de los ochenta a través de un sugerente análisis de los mensajes de los medios de comunicación de masas. Según esta autora, el mensaje de la reacción antifeminista se mantiene en dos pilares ideológicos falsos pero machaconamente repetidos: 1) la igualdad sexual ya es un hecho, el feminismo, es cosa del pasado, y 2) la igualdad sexual ha empobrecido y estresado la vida de las mujeres, las ha hecho más infelices. Frente a esta reacción ideológica contra el feminismo las últimas campañas contra la violencia de género pueden interpretarse, siguiendo la terminología de McAdam, como una oportunidad cultural para difundir el feminismo (McAdam, 1994). Es decir, como una plataforma para dramatizar ante la opinión pública una contradicción manifiesta entre un valor cultural cada vez más aceptado, como es la igualdad sexual, y su falta de concreción real, como muestra el recurso al uso de la violencia para controlar el comportamiento de las mujeres. 


\section{RIS}

\section{REFERENCIAS BIBLIOGRÁFICAS}

AGUILAR, S. (2001), "Movimientos sociales y cambio social”, Revista Internacional de Sociologia $(R I S), \mathrm{n}^{\circ} 30$, pp. 29-62.

ALBERDI, I. (1999), "El significado del género en las ciencias sociales", Politica y Sociedad, $\mathrm{n}^{\circ}$ 32, pp. 9-21, Universidad Complutense de Madrid.

ALBERDI, I. y N. MATAS (2002), La violencia doméstica. Informe sobre los malos tratos a mujeres en España, Barcelona, Fundación La Caixa. (Edición electrónica disponible en www.estudios.lacaixa.es).

AMORÓS, C. (1998), Tiempo de feminismo, Madrid, Cátedra.

(1990), "Violencia contra las mujeres y pactos patriarcales", en V. Maquieira y C. Sánchez (comps.) Violencia y sociedad patriarcal, Madrid, Pablo Iglesias.

ASTELARRA, J. (dir.) (1990), Participación política de las mujeres, Madrid, CIS/Siglo XXI.

BECK, U., A. GIDDENS y S. LASH (1997), Modernización reflexiva, Madrid, Alianza Universidad.

BOLANCÉ, J. y C. LÍAN (comps.) (2002), Violencia, género y coeducación, Córdoba, Ayuntamiento de Córdoba.

BORDERÍAS, C., C. CARRASCO y C. ALEMANY (comps.) (1994), Las mujeres y el trabajo. Rupturas conceptuales, Barcelona, Icaria.

BOURDIEU, P. (2000), La dominación masculina, Barcelona, Anagrama.

BROWNMILLER, S. (1976), Against our Will, Harmondsworth, Penguin Books.

CASTELLS, M. (1998), La era de la información. Vol. 2 El poder de la identidad, Madrid, Alianza.

CENTRO DE INVESTIGACIONES SOCIOLÓGICAS (2001), La violencia doméstica, Encuesta, Estudio 2.411.

COMISIÓN EUROPEA (1999), Los europeos/as y sus opiniones sobre la violencia doméstica contra las mujeres, Eurobarómetro.

CONSEJERIAA DE LA MUJER DE LA COMUNIDAD DE MADRID (2001), Propuestas de las Asociaciones de Mujeres para un Plan Integral contra la violencia hacia las Mujeres, Madrid.

DEFENSOR DEL PUEBLO (1998), Violencia contra las mujeres, Madrid, Oficina del Defensor del Pueblo.

DURÁN, M.A. (ed.) (1996), Mujeres y hombres en la formación de la teoria sociológica, Madrid, Centro de Investigaciones Sociológicas. 
(dir.) (1988), De puertas adentro, Madrid, Instituto de la Mujer.

ESCARIO, P., I. ALBERDI y A.I. LOPEZ-ACOTTO (1996), Lo personal es politico. El movimiento feminista en la transición, Madrid, Instituto de la Mujer.

EYERMAN, R. y A. JAMISON (1991), Social Movements. A Cognitive Approach, Pensilvania, The Pennsylvania State University Press.

FAGOAGA, C. (1999), La violencia en los medios de comunicación. Maltrato a la pareja y agresión sexual, Madrid, Dirección General de la Mujer de la Comunidad Autónoma de Madrid.

FALUDI, S. (1993), Reacción, Barcelona, Anagrama.

FERNÁNDEZ BUEY, F. y J. RIECHMANN (1994), Redes que dan libertad, Barcelona, Paidós.

FERRER, V. y E. BOSCH (2002), La voz de las invisibles, Madrid, Cátedra.

(2000), "La violencia de género: de cuestión privada a problema social", Revista de Intervención Psicosocial, vol. 9, $\mathrm{n}^{\circ} 1$.

FISAS, V. (ed.) (1998), El sexo de la violencia, Barcelona, Icaria.

FRASER, N. (1997), Iustitia Interrupta, Bogotá, Siglo del Hombre Editores.

FUNES, M.J. y R. ADELL (eds.) (2003), Movimientos sociales: cambio social y participación, Madrid, UNED, Colección Varia.

GELB, J. (1992), "Feminismo y acción política”, en J.D. Russel y M. Kuechler (comps.), Los nuevos movimientos sociales: un reto al orden politico, Valencia, Alfons el Magnànim.

GOFFMAN, E. (1974), Frame Analysis, Boston, Northeastern University Press.

GUSFIELD, J. (1994), “La reflexividad de los movimientos sociales”, E. Laraña y J. Gusfieled (coords.), Los nuevos movimientos sociales, Madrid, CIS.

HARDING, S. (1991), Whose Science? Whose Knowledge?, Ithaca, Cornell University Press.

IBARRA, P. y B. TEJERINA (coords.) (1998), Los Movimientos Sociales, Madrid, Trotta.

INSTITUTO ANDALUZ DE LA MUJER (1999), Como tratar bien los malos tratos: Manual de estilo para los medios de comunicación, Sevilla.

INSTITUTO DE LA MUJER (1998), Prevenir la violencia. Una cuestión de cambio de actitud, Cuaderno de Educación, $n^{\circ} 7$, Madrid.

(1998), Plan Integral contra la violencia doméstica, Madrid.

(2000), Las mujeres en cifras, http://www.mtas.es/mujer/mcifras/principal.htm 
RIS

REVISTA INTERNACIONAL DE SOCIOLOGIA

(2001), II Plan Integral contra la violencia doméstica, Madrid, Instituto de la Mujer.

IZQUIERDO, M.J. (1998), El malestar en la desigualdad, Madrid, Cátedra.

JAVALOY, F., A. RODRÍGUEZ y E. ESPELT (2001), Comportamiento colectivo y movimientos sociales, Madrid, Prentice Hall.

JOHNSTON, H. y B. KLANDERMANS (eds.) (1995), Social Movements and Culture, Londres, UCL Press.

JÓNASDÓTTIR, A.G. (1993), El poder del amor, Madrid, Cátedra.

JORNADAS FEMINISTAS CÓRDOBA (comp.) (2000), Feminismo. es ... y será, Córdoba, Servicio de Publicaciones Universidad de Córdoba.

LARAÑA, E. (1999), La construcción de los movimientos sociales, Madrid, Alianza Editorial.

LARAÑA, E. y J. GUSFIELD (coords.) (1994), Los Nuevos Movimientos Sociales, Madrid, Centro de Investigaciones Sociológicas (CIS)

LARRAURI, E. (comp.) (1994), Mujeres, Derecho penal y criminologia, Madrid, Siglo XXI.

(1991), La herencia de la criminología crítica, Madrid, Siglo XXI.

LENGERMANN, P.M. y J. NIEBRUUGGE-BRANTLEY (1993), "Teoría feminista contemporánea", en G. Ritzer (ed.), Teoria Sociológica Contemporánea, Madrid, McGraw-Hill.

LOZOYA, J.A. (1999), "Violencia masculina contra las mujeres", Programa: Hombres por la igualdad, Delegación de Salud y Género, Ayuntamiento de Jerez.

MAQUIEIRA, V. y C. SÁNCHEZ (comps.) (1990), Violencia y sociedad patriarcal, Madrid, Pablo Iglesias.

MARTÍN, E. (1999), Las violencias cotidianas: cuando las victimas son las mujeres, Madrid, Instituto de la Mujer.

MATUD, M.P., R.J. MARRERO, M. CARBALLEIRA, O. MORAZA y L. AGUILERA (2003), "Mujeres maltratadas por su pareja: atribuciones causales y dinámica de la violencia", Clepsydra, $\mathrm{n}^{\circ} 2$, pp. 57-69.

McADAM, D. (1994), “Cultura y movimientos sociales”, en E. Laraña y J. Gusfieled (eds.), Los nuevos movimientos sociales, Madrid, CIS.

(1982), Political Process and the Development of Black Insurgency, Chicago, University of Chicago Press.

McADAM, D., J.D. McCARTHY y M.N. ZALD (1999), Movimientos sociales: perspectivas comparadas, Madrid, Istmo. 
MELUCCI, A. (1994), “¿Qué hay de nuevo en "los nuevos movimientos sociales”, en E. Laraña y J. Gusfieled (eds.), Los nuevos movimientos sociales, Madrid, CIS.

(1989), Nomads of the present, Filadelfia, Temple University Press.

MIEDZIAN, M. (1995), Chicos son, hombres serán, Madrid, horas y Horas.

MIGUEL ÁLVAREZ,A. de (1995), “Género y conducta desviada: estado de la cuestión” en C. Brullet y P. Carrasquer (coords.), Sociologia de las relaciones de género, Madrid, Instituto de la Mujer.

(1998), "La redefinición de lo público y lo privado", Actas del V Congreso Estatal de Intervención Social, Madrid, Ministerio de Trabajo y Asuntos Sociales.

(1999), "La sociología olvidada", Política y Sociedad, n 32, Universidad Complutense de Madrid, pp. 161-171.

(2002), "Hacia un nuevo contrato social", en J.M. Robles (coord.), El reto de la participación. Movimientos y organizaciones: una panorámica comparativa, Madrid, Antonio Machado Eds.

MILLETT, K. (1995), Politica sexual, Madrid, Cátedra.

MOLINA, C. (1994), Dialéctica feminista de la Ilustración, Barcelona, Anthropos.

MORRIS, A.D. y C.M. MUELLER (1992), Frontiers in Social Movements Theory, New Haven, Yale University Press.

MUELLER, C.M. (1994), "Identidades colectivas y redes de conflicto", en E. Laraña y J. Gusfield (coords.), Los nuevos Movimientos Sociales, Madrid, CIS.

MULLENDER, A. (2000), La violencia domestica... una nueva visión de un viejo problema, Barcelona, Paidós.

MURILLO, S. (2002), "Agresiones contra las mujeres", La situación social de las mujeres en Andalucia, Sevilla, Instituto de la Mujer.

(2000), Relaciones de poder entre hombres y mujeres, Madrid, FMP y Ministerio de Trabajo y Asuntos Sociales.

OSBORNE, R. (coord.) (2001), La violencia contra las mujeres. Realidad social y politicas públicas, Madrid, UNED Ediciones.

PÉREZ DEL CAMPO, A.M. (1996), Una cuestión incomprendida: el maltrato a la mujer, Madrid, Ediciones horas y horas.

PATEMAN, C. (1995), El contrato sexual, Barcelona, Anthropos.

POSADA, L. (2001), “'Las hijas deben ser siempre sumisas' (Rousseau). Discurso patriarcal y violencia contra las mujeres: reflexiones desde la teoria feminista”, en A. Bernárdez (ed.) Violencia de género y sociedad: una cuestión de poder, Madrid, Ayuntamiento de Madrid, Instituto de 


\section{RIS}

Investigaciones Feministas (UCM).

PULEO, A. (1995), "Patriarcado", en C. Amorós (dir.), Diez palabras clave sobre mujer, Pamplona, Verbo Divino.

REVILLA, M. (1994), "El concepto de movimiento social: acción, identidad y sentido", Zona Abierta, $\mathrm{n}^{\circ}$ 69, pp. 181-213.

REX, J. (1985), El conflicto social, Madrid, Siglo XXI.

ROBLES MORALES, J.M. (coord.) (2002), El reto de la participación. Movimientos y organizaciones: una panorámica comparativa, Madrid, Antonio Machado Eds.

SABUCEDO, J.M., J. GROSSI y C. FERNÁNDEZ (1998), “Los Movimientos Sociales y la creación de un sentido común alternativo”, en P. Ibarra y B. Tejerina (coords.).

SUBIRATS, M. (1998), Con diferencia, Barcelona, Icaria.

SALTZMAN, J. (1992), Equidad y género, Madrid, Cátedra.

SWIDLER, A. (1986), “Culture in Action: Symbols and Strategics”, American Sociological Review, 51, pp. 273-286.

TAYLOR, I., P. WALTON y J. YOUNG (1977), La nueva criminologia, Buenos Aires, Amorrortu.

THEMIS (2000), La respuesta penal a la violencia contra las mujeres, Madrid, Consejo de la Mujer de la Comunidad de Madrid.

VALCÁRCEL, A. (1997), La politica de las mujeres, Madrid, Cátedra.

(1991), Sexo y Filosofia, Barcelona, Anthropos.

VALIENTE, C. (1996), "Partial achievements of central-state public policy against violence against women in post-authoritarian Spain", en C. Borin (ed.), Women in a Violent World, Edimburgo, Edinburgh University Press.

VARELA, N. (2002), Íbamos a ser reinas. Mentiras y complicidades que sustentan la violencia contra las mujeres, Barcelona, Ediciones B. 